\title{
Use Value of Food Plants in the Xi'iuy Indigenous Community of Las Guapas, Rayon, San Luis Potosi, Mexico
}

\author{
Haydeé Carbajal-Esquivel, Javier Fortanelli Martínez, ${ }^{1}$ José García-Pérez, Juan A. Reyes-Agüero, Laura \\ Yáñez-Espinosa, Mark Bonta.
}

Author Addresses: ${ }^{1}$ Instituto de Investigación de Zonas Desérticas, Universidad Autónoma de San Luis Potosí, San Luis Potosí, SLP, México fortanel@uaslp.mx

Received: October 31, 2011

Volume 3:39-55

Published: August 21, 2012

(C) 2012 Society of Ethnobiology

Abstract: Native communities' erosion of ethnobotanical knowledge of food plants is a global concern. This investigation focuses on a Xi'iuy community in the Sierra Madre Oriental, San Luís Potosí, México. A total of 21 randomly-selected families participated (22\% of the total population). The 56 people who were interviewed-an average of 2.7 per family-- were separated into four groups (fathers, mothers, single sons, single daughters). To investigate the use value of each plant, a collection of 54 food specimens was shown to the informants. Knowledge of each food species' uses was compared between genders and age groups. The results included figures that were lower than expected, as well as less knowledge among women than men, particularly among underage daughters. The difference in use value between men and women in this community is explicable by cultural factors: i.e., women's participation in agriculture and plant collecting is minimal. This, along with men's seasonal migration for work (men are usually wage laborers half the year in the sugarcane harvest, and the other half they cultivate their own land), plus increasing availability of commercial food in grocery stores, contributes to the steady loss of ethnobotanical knowledge.

Key words: Use value, food plants, quantitative ethnobotany, Xi'iuy ethnic group.

\section{Introduction}

The geographical region known as La Pamería, located in the Sierra Madre Oriental, in the states of San Luis Potosí and Querétaro, México (Chemin, 1984; Álvarez, 1996; Vázquez, 2010), has evolved through a lengthy and complex historical processes involving the Xi'iuy ethnic group (known by mestizos as "Pame", thus "La Pamería" means "land of the Pames"). State policies over the centuries have favored the reduction of Xi'iuy territory and displacement of its people, resulting in land and water resources becoming increasingly concentrated in the hands of mestizo and Criollo landowners (Ordóñez 2004). From the $16^{\text {th }}$ to the $18^{\text {th }}$ centuries, the Xi'iuy people, facing long-lasting conflicts with encomienda holders, hacienda owners, and bellicose, nomadic tribes, retreated to the most isolated and rugged parts of the Sierra Madre, where they eked out an existence as smallholders (Velázquez 1987).

Although Xi'iuy land contains abundant resources and high productive potential, it is characterized by stark poverty. Chronic malnutrition, a poverty-related condition, is rife, and can be explained by several factors: inadequate agricultural and food-gathering strategies; dietary changes resulting from the introduction of commercial food products of little nutritional value; adoption of alien cultural mores fomented by temporary, local emigration (Anonymous 1999). Ethnographic research carried out in two nearby indigenous towns ( $\mathrm{La}$ Manzanilla and Agua Puerca) highlighted a similar situation (Cotonieto, 2011). During the dry season, males 15 years and above typically labor in the sugarcane harvest, in nearby intermontane valleys. During this time, they return each weekend to their homes. In the rainy season, they plant and harvest maize and beans on their own land in the mountains.

This situation appears to favor continuous erosion of Xi'iuy knowledge of local food resources. A concept that contributes to the understanding of this issue is use value, i.e., the capability of a given resource, good, or service to meet the needs of an individual or society (Callan and Thomas 1996; Asafu 2005). In the case of plants, a given species will have a high use value if a relatively elevated number of consumers or users 
make use of it for a wide variety of purposes. Hence, this study analyzes the use value of local food plants by gender and age groups in a Xi'iuy community.

\section{Materials and Methods}

This study was conducted from January 2006 to April 2007 in the village of Las Guapas, municipality of Rayón, San Luis Potosí state, Mexico (99²7'40'W; $21^{\circ} 55^{\prime} 45^{\circ} \mathrm{N}$; 1080 m.a.s.l.) (Anonymous 1980). Las Guapas comprises 96 families whose livelihoods include subsistence agriculture, small-scale commerce in fruits and vegetables, cattle-raising, fabrication and sale of handicrafts, and agricultural labor outside the community. Las Guapas has a warm climate and is located in a valley with reddish-brown, clayey soils derived from fine-grained sedimentary rock. It is flanked by limestone hills that reach altitudes of nearly 1400 meters above sea level. The dominant vegetation type is Quercus oak forest.

First, we conducted a community meeting to seek consent for the research. Informed consent was granted. The sample included 21 families $(21.8 \%$ of the total number of families in Las Guapas, ) selected at random. Then, we conducted a field survey, using data from local informants along with botanical identification, in forests, lands under cultivation, and community orchards. We identified 159 species of ethnobotanical interest and nine different uses. Thenceforth, following the objective of this study, we made a collection of 54 specimens with uses for food, and 12 specimens with non-food uses. Only $71 \%$ of food plants collected were used in this study (the remaining $29 \%$ were unidentifiable owing to the poor state of the specimens, and were thus discarded), to avoid potential species misidentifications due to informants' fatigue and/or boredom toward the end of the study. This method is superior to that employed in a study by Lyen y Nguyen (2003) in which, to avoid fatigue and loss of interest on the part of the informants, just ten photographs were used to evaluate traditional fruit and vegetable knowledge among Vietnamese in Vietnam and Hawaii.

After we gathered data on plants collected, we calculated use values following Phillips and Gentry (1993a, b). This method allows researchers to assess 1) the ability of interviewees to recognize plant species and 2) interviewees' knowledge of their uses. We showed interviewees plant specimens and asked them to describe different uses. We ran tests on these data to assess the importance of a given species based on its various uses (see Table 1 for equations used). We also investigate interviewees' knowledge of the species' common name, Xi'iuy name, part of the plant used and means of preparation, and frequency of inclusion in the diet.

We interviewed 21 female heads of family, 11 male heads of family (it was impossible to interview all male heads of family in the sample due to their temporary absence from the community for work purposes), and single ("underage") sons and daughters between 12 and 22 years of age (seven sons and seven daughters). We made the assumption, based on Chemin (1984) and Cotonieto (2010) that persons in the 12-to-22 age group had already gained sufficient knowledge of community life and traditions to apply in the future as heads of family. To avoid confusion between interviewees, we conducted separate and isolated sessions for each interviewee.

\section{Results and Discussion}

Results obtained via the equations shown in Table 1 allowed us to analyze differential knowledge of plant uses among the Xi'iuy people. Assuming that only plants used as food were included, our minimum expectation was that the interviewee would be able to identify at least one type of food produced from each plant sample. However, some interviewees faced difficulties in identifying specimens, particularly for species belonging to the same taxonomical family, or did not know of specific alimentary uses at all.

Table 2 displays some of the species collected (refer to Appendix 1 for the complete list) to demonstrate how use values were calculated. The example shows the outcome of interviews with 11 heads of family. It is evident that Erythrina coralloides has a maximum $\mathrm{Vt}$ of 4.0, meaning that interviewees acknowledged that this species is used both as food and for other purposes. Although the table lists only a fraction of all species considered, complete results for these species are displayed. E. coralloides has the highest use value relative to all others $(\mathrm{Vsp}=2.73)$, as a result of the number of distinct uses associated with this species. Interviewee 1 in the adult men group assigned the highest figures to the set of plants analyzed ( Vs = 1.17); this was likely due to his age: at 60 years old, he was the oldest of the 11 interviewees in his group.

Table 3 displays 25 species included in the plant collection and the respective total use value for each interviewee group. Use values (Vs) were compared among interviewee groups through the U-MannWhitney test. From this, only differences between adult men and underage women (Vppa versus Vpja), and 
between underage men and underage women (Vpjo versus Vspja), were statistically significant $(\mathrm{U}=39.5$, $\mathrm{p}$ $<0.01 ; \mathrm{U}=21, \mathrm{p}<0.01$, respectively). This may be correlated with the custom of women's visiting forests and parcels only when accompanying their husbands or sons. As a result, women access to knowledge is limited relative to men's. Nevertheless, when a woman becomes the head of household (because her partner has either died or emigrated to the US or to a distant Mexican state), she learns to use the different plants as food and also identifies other uses.

Xi'iuy women's inferior knowledge of plant uses contrasts sharply with the situation of a mestizo Mapuche community in Neuquen, Argentina (Lozada et al. 2006), where women play the leading role in preserving ethnobotanical knowledge, and no significant differences between men and women vis-àvis knowledge of plant uses are reported. In a similar context, Hadza women in South Africa walk some eight $\mathrm{km}$ to collect water as well as fruits and other food plants (Youngblood 2004). More restrictive situations exist among Bribri and Cabecar communities in Costa Rica, where women are barred from utilizing certain plants in anthropic landscapes (Ramos and Del Monte 2004). The closest resemblance to the situation of the Xi'iuy people of Las Guapas is Santa Isabel Chalma, Amecameca, Mexico, a community involved primarily in forest exploitation. There, men possess a deeper ethnobotanical knowledge than women; working in the forest is said to be an activity unsuitable for unmarried women (Estrada 1996). It is only when married that a woman learns knowledge about plants from her husband. In the Cuenca del Caura, Venezuela, Souto and Ticktin (2012) obtained similar results in a study that showed that men know more edible wild fruits than women, and elderly women know more plants-particularly those found close to dwellingsthan younger women.

Within a similar context, the difference between Vpjo and Vspja in Las Guapas is due to the fact that boys begin their acquaintance with plant uses at an earlier age than girls, as they are afforded the opportunity to visit the forest with their fathers. Furthermore, the difference in $\mathrm{Vp}$ between fathers and sons should be noted: sons easily identified species and described at least their uses as food, while fathers faced various problems in identifying them. This may be due to the fact that fathers have lost regular contact with plants as a result of constant, temporary emigration for work. These findings are cause for great concern, because issues involving transmission of knowledge derived from traditional differential gender roles are exacerbated by the ongoing loss of contact with environmental resources and growing economic and cultural pressures to consume processed, commercial foods readily available in local grocery stores. In this respect, some authors have pointed out diverse tendencies in relation to increased contact with the outside. For example, Hamlin and Salick (2003), working in the Peruvian Amazon, found that Yanesha communities were not negatively affected by the opening of a modern highway. Although it brought more people to a previous isolated zone and augmented the presence of commercial activities, the Yanesha were able to adapt by not only enriching their dooryard gardens and diets with new flavors and ingredients, but also maintaining their traditional agricultural knowledge. Similarly, McMillen (2012) found in Tanga, Tanzania, that medicinal plant knowledge was being improved rather that eroded with better connections to regional markets. By contrast, Voeks and Leony (2004) showed that the process of modernization in Lençóis, Eastern Brazil, is incompatible with the persistance of ethnobotanical knowledge of medicinal plants, because there is a positive relationship between illiteracy and local traditional knowledge.

In Las Guapas, forest plant species with high use values included "bignerón" (Ficus cotinifolia Kunth) and avocado (Persea americana Mill.). These species are also found in household orchards, but they were classified as forest plants because they also grow wild. Cultivated plants with highest use values were "teja corn" (Helianthus annuus L.) and "eparote" (Chenopodium ambrosioides L.). The species with highest use value in orchards was "patol" (Erythrina coralloides DC.): as already mentioned, this species is utilized in a number of different ways. Another relevant plant in typical Xi 'iuyky orchards is "ruda" (Ruta chalepensis L.), a species used both as medicine and spice.

Table 3 shows that plants with the highest use values (for example, Cnidoscolus multilobus and Conostegia xalapensis) correspond mostly to either anthropic landscapes like orchards and plots under cultivation, or to disturbed vegetation. In general, similar uses for food occur across different environments; based on the U Mann-Whitney test, there are no statistically significant differences between food plants from forests and from orchards in terms of: a) frequency of consumption (number of days per year that a given species is consumed by the family interviewed) ( $\mathrm{U}=$ 
136.5, $\mathrm{p}>0.05)$, and $\mathrm{b})$ the plant's use value $(\mathrm{U}=136$, $\mathrm{p}>0.05)$.

\section{Conclusions}

Use value figures for plant species consumed as food were lower than expected, with extent of knowledge below average among underage daughters and above average among underage sons. Differences in use value between men and women are most likely related to a cultural context in which women participate in agriculture and plant collection in only a limited fashion.

Plants with the highest use values correspond to anthropic landscapes such as orchards and cultivated fields, and to areas with disturbed vegetation. In terms of frequency of consumption or use value, plants collected in the forest displayed no significant differences relative to plants grown in orchards.

\section{References Cited}

Álvarez C. H. 1996. Problemática agraria en la pamería potosina: panorama actual. In: L. Torre (Coord.) Xi'Oi Coloquio pame. Los pames de San Luis Potosí y Querétaro. pp. 159-170. Centro de Investigaciones Históricas de San Luis Potosí and Instituto de Cultura de San Luis Potosí, San Luis Potosí, México.

Anonymous. 1980. Tamasopo. Carta topográfica. F-14-C18, Escala 1:50000. Dirección General de Geografía del Territorio Nacional, México.

Anonymous. 1999. Diagnóstico socioeconómico, productivo y de análisis económico financiero de proyectos tipo en la zona pame de San Luis Potosí. Programa de Desarrollo Productivo Sostenible en Zonas Rurales Marginadas. SAGARPA, San Luis Potosí, México.

Asafu-Adjaye, J. 2005. Environmental Economics for Noneconomists. Techniques and Policies for Sustainable Development. World Scientific Publishing, Singapore, Malaysia.

Callan, S. J. and J. M. Thomas. 1996. Environmental and Management Theory, Policy and Applications. Irwin McGraw-Hill, Chicago, Illinois.

Bassler, H. C. 1984. Los pames septentrionales de San Luis Potosí. Instituto Nacional Indigenista, México.

Santilez, H. C. 2011. No tenemos las mejores tierras ni vivimos en los mejores pueblos... pero acá seguimos: Ritual agricola, organización social y cosmovisión de los pames del norte. El Colegio de San Luis, San Luis Potosí, México.

Estrada, M. E. 1996. Etnobotánica forestal en Santa Isabel Chalma, Amecameca, México. Unpublished
Master's Thesis, Department of Botany, Colegio de Postgraduados Montecillo, Texcoco, Estado de México.

Hamlin, C. C. and J. Salick. 2003. Yanesha Agriculture in the Upper Peruvian Amazon: Persistence and Change Fifteen Years Down the "Road". Economic Botany 57:163-180.

Lien, M. and T. Nguyen. 2003. Comparison of food plant knowledge between urban Vietnamese living in Vietnam and in Hawai'i. Economic Botany 57:472-480.

Lozada, M., Ladio A., and M. Weigandt.

2006. Cultural transmission of ethnobotanical knowledge in a rural community of Northwestern Patagonia, Argentina. Economic Botany 60:374-385.

McMillen, H. 2012. Ethnobotanical Knowledge Transmission and Evolution: The Case of Medicinal Markets in Tanga, Tanzania. Economic Botany 20:1-11.

Ordóñez, C. G. 2004. Pames. Programa de las Naciones Unidas para el Desarrollo, México City, México.

Phillips, O. and A. H. Gentry. 1993a. The useful plants of Tambopata, Perú: I. Statistical hypotheses tests with a new quantitative technique. Economic Botany 47:15-32.

Phillips, O. and A. H. Gentry. 1993b. The useful plants of Tambopata, Perú: II. Additional hypothesis testing in quantitative ethonobotany. Economic Botany 47:33-43.

Ramos, G. S. C. and J. P. Del Monte. 2004. The use of tropical forest (agroecosystems and plant harvesting) as a source of food in the Bribri and Cabecar Cultures in the Coast of Costa Rica. Economic Botany 58:58-71.

Souto, T. and T. Ticktin. 2012. Understanding Interrelationships among Predictors (Age, Gender, and Origin) of Local Ecological Knowledge. Economic Botany 20:1-16.

Vázquez E. A. 2010. Xi'oi Los verdaderos hombres. Atlas etnográfico pames de la Sierra Gorda Queretana. Universidad Autónoma de Querétaro, Qurétaro, México.

Velázquez, P. F. 1987. Colección de documentos para la historia de San Luis Potosí. Archivo Histórico del Estado de San Luis Potosí, San Luis Potosí, México.

Voeks, R. A. and A. Leony. 2004. Forgetting the Forest: Assessing Medicinal Plant Erosion in Eastern Brazil. Economic Botany 58:S294-S306.

Youngblood, D. 2004. Identification and quantification of edible plant foods in the Upper (Nama) Karoo, South Africa. Economic Botany 58:S43-S65. 
Biosketch

Haydeé Carbajal-Esquivel is a biologist with a Master's Degree in Environmental Science from the Autonomous University of San Luis Potosi in Mexico. He is also a High School Director at IUEM University in Mexico. 
Table 1. Equations used to calculate use value of plant species [modified from original equations by Phillips and Gentry (1993a,b)].

\begin{tabular}{lll}
\hline \multicolumn{1}{c}{ Feature } & \multicolumn{1}{c}{ Index } & \multicolumn{1}{c}{ Definition } \\
\hline $\begin{array}{l}\text { Use value per interviewee for } \\
\text { each species }\end{array}$ & $\begin{array}{l}\mathrm{V}_{\mathrm{t}}=\mathrm{Number} \text { of uses of each } \\
\text { species known to given } \\
\text { interviewee }\end{array}$ & $\begin{array}{l}\text { Total number of uses of each species: } \\
\mathrm{V}_{\mathrm{t}}=0, \mathrm{~V}_{\mathrm{t}}=1 \text {, etc. }\end{array}$ \\
Mean use value per interviewee & $\begin{array}{l}\mathrm{V}_{\mathrm{s}}=\Sigma \mathrm{V}_{\mathrm{t}} / \text { Total number of } \\
\text { species observed per } \\
\text { interviewee }\end{array}$ & $\begin{array}{l}\text { Average use value per interviewee for } \\
\text { each species observed. }\end{array}$ \\
Mean use value per species & $\begin{array}{l}\mathrm{V}_{\mathrm{sp}}=\Sigma \mathrm{V}_{\mathrm{t}} / \text { number of } \\
\text { interviewees that observed the } \\
\text { plant species }\end{array}$ & $\begin{array}{l}\text { Average use value assigned to a given } \\
\text { species by all interviewees. }\end{array}$ \\
Use value of food species per \\
group of interviewees
\end{tabular}


Table 2. Use values of food plants in Las Guapas by heads of family.

\begin{tabular}{|c|c|c|c|c|c|c|c|c|c|c|c|c|}
\hline \multirow[b]{2}{*}{ Interviewee } & \multirow[b]{2}{*}{1} & \multicolumn{11}{|c|}{ Number of uses recognized per interviewee $\left(V_{t}\right)$} \\
\hline & & 2 & 3 & 4 & 5 & 6 & 7 & 8 & 9 & 10 & 11 & Vsp \\
\hline \multicolumn{13}{|l|}{ Species } \\
\hline Erythrina coralloides & 4 & 3 & 2 & 2 & 4 & 4 & 3 & 1 & 4 & 2 & 1 & 2.73 \\
\hline Ficus cotinifolia & 3 & 4 & 2 & 2 & 1 & 4 & 3 & 2 & 2 & 2 & 1 & 2.36 \\
\hline Persea americana. & 2 & 4 & 2 & 2 & 1 & 1 & 3 & 1 & 3 & 1 & 1 & 1.91 \\
\hline Chenopodium ambrosioides & 1 & 1 & 1 & 1 & 1 & 2 & 1 & 1 & 1 & 2 & 1 & 1.18 \\
\hline Helianthus annuus & 2 & 2 & 1 & 1 & 1 & 1 & 1 & 1 & 1 & 1 & 1 & 1.18 \\
\hline Psidium guajava & 2 & 1 & 1 & 1 & 1 & 1 & 1 & 1 & 2 & 1 & 1 & 1.18 \\
\hline Cnidosculus multilobus & 2 & 1 & 1 & 1 & 1 & 1 & 1 & 1 & 1 & 1 & 1 & 1.09 \\
\hline Coffea arabica & 1 & 2 & 1 & 1 & 1 & 1 & 2 & 1 & 1 & 1 & 0 & 1.09 \\
\hline Amaranthus bybridus & 1 & 1 & 1 & 1 & 1 & 1 & 1 & 1 & 1 & 1 & 1 & 1.00 \\
\hline Baubinia chapulbuacania & 1 & 1 & 1 & 1 & 1 & 1 & 1 & 1 & 1 & 1 & 1 & 1.00 \\
\hline Carica papaya & 1 & 1 & 1 & 1 & 1 & 1 & 1 & 1 & 1 & 1 & 1 & 1.00 \\
\hline Carya ovata var. mexicana & 1 & 1 & 1 & 1 & 1 & 1 & 1 & 1 & 1 & 1 & 1 & 1.00 \\
\hline Conostegia xalapensis & 1 & 1 & 1 & 1 & 1 & 1 & 1 & 1 & 1 & 1 & 1 & 1.00 \\
\hline Gonolobus niger & 1 & 1 & 1 & 1 & 1 & 1 & 1 & 1 & 1 & 1 & 1 & 1.00 \\
\hline Juglans mollis & 1 & 1 & 1 & 1 & 1 & 1 & 1 & 1 & 1 & 1 & 1 & 1.00 \\
\hline \multicolumn{13}{|l|}{ Lycopersicon esculentum var. } \\
\hline cerasiforme & 1 & 1 & 1 & 1 & 1 & 1 & 1 & 1 & 1 & 1 & 1 & 1.00 \\
\hline Mangifera indica & 1 & 1 & 1 & 1 & 1 & 2 & 1 & 1 & 1 & 0 & 1 & 1.00 \\
\hline Manihot esculenta & 1 & 1 & 1 & 1 & 1 & 1 & 1 & 1 & 1 & 1 & 1 & 1.00 \\
\hline Musa x paradisiaca & 1 & 1 & 1 & 1 & 1 & 1 & 1 & 1 & 1 & 1 & 1 & 1.00 \\
\hline Nopalea cochenillifera & 1 & 1 & 1 & 1 & 1 & 1 & 1 & 1 & 1 & 1 & 1 & 1.00 \\
\hline Opuntia sp. & 1 & 1 & 1 & 1 & 1 & 1 & 1 & 1 & 1 & 1 & 1 & 1.00 \\
\hline Phaseolus coccineus & 1 & 1 & 1 & 1 & 1 & 1 & 1 & 1 & 1 & 1 & 1 & 1.00 \\
\hline Phaseolus vulgaris & 1 & 1 & 1 & 1 & 1 & 1 & 1 & 1 & 1 & 1 & 1 & 1.00 \\
\hline Ruta chalepensis & 1 & 1 & 1 & 1 & 1 & 1 & 1 & 1 & 1 & 1 & 1 & 1.00 \\
\hline Saccharum officinarum & 1 & 1 & 1 & 1 & 1 & 1 & 1 & 1 & 1 & 1 & 1 & 1.00 \\
\hline Tagetes filifolia & 1 & 1 & 1 & 1 & 1 & 1 & 1 & 1 & 1 & 1 & 1 & 1.00 \\
\hline Vigna unguiculata & 1 & 1 & 1 & 1 & 1 & 1 & 1 & 1 & 1 & 1 & 1 & 1.00 \\
\hline Zingiber officinale & 1 & 1 & 1 & 1 & 2 & 1 & 1 & 1 & 1 & 1 & 0 & 1.00 \\
\hline Canna indica & 1 & 1 & 2 & 1 & 1 & 0 & 1 & 1 & 1 & 0 & 1 & 0.91 \\
\hline Curcuma longa & 1 & 1 & 1 & 1 & 1 & 1 & 1 & 1 & 1 & 1 & 0 & 0.91 \\
\hline Cymbopogon citratus & 1 & 1 & 1 & 1 & 1 & 1 & 1 & 1 & 1 & 0 & 1 & 0.91 \\
\hline Pachyrbizus erosus & 1 & 1 & 1 & 1 & 1 & 1 & 1 & 1 & 1 & 0 & 1 & 0.91 \\
\hline Phytolacca icosandra & 1 & 1 & 1 & 1 & 1 & 1 & 1 & 1 & 1 & 1 & 0 & 0.91 \\
\hline Sechium edule & 1 & 1 & 2 & 1 & 1 & 1 & 1 & 1 & 1 & 0 & 0 & 0.91 \\
\hline Tigridia pavonia & 1 & 1 & 1 & 1 & 1 & 1 & 1 & 1 & 1 & 1 & 0 & 0.91 \\
\hline Capsicum anпuиm & 1 & 0 & 1 & 1 & 1 & 1 & 0 & 1 & 1 & 1 & 1 & 0.82 \\
\hline Capsicum annuum var. aviculare & 1 & 0 & 1 & 1 & 1 & 1 & 0 & 1 & 1 & 1 & 1 & 0.82 \\
\hline Jatropha curcas & 1 & 1 & 0 & 1 & 1 & 1 & 1 & 0 & 1 & 1 & 1 & 0.82 \\
\hline Pisum sativum & 1 & 0 & 1 & 1 & 1 & 1 & 0 & 1 & 1 & 1 & 1 & 0.82 \\
\hline Syngonium podophyllum & 1 & 0 & 1 & 1 & 1 & 1 & 0 & 1 & 1 & 1 & 1 & 0.82 \\
\hline Arachis bypogaea & 1 & 1 & 1 & 1 & 1 & 0 & 1 & 1 & 1 & 0 & 0 & 0.73 \\
\hline Citrus maxima & 1 & 1 & 1 & 1 & 1 & 0 & 1 & 1 & 1 & 0 & 0 & 0.73 \\
\hline Morus aff. celtidifolia & 1 & 1 & 1 & 1 & 1 & 0 & 1 & 1 & 1 & 0 & 0 & 0.73 \\
\hline Physalis philadelphica & 2 & 0 & 0 & 1 & 2 & 0 & 0 & 0 & 2 & 1 & 0 & 0.73 \\
\hline
\end{tabular}




\begin{tabular}{|c|c|c|c|c|c|c|c|c|c|c|c|c|}
\hline Casimiroa edulis & 1 & 1 & 0 & 1 & 1 & 0 & 0 & 0 & 1 & 1 & 1 & 0.64 \\
\hline Citrus aurantifolia & 1 & 1 & 1 & 1 & 0 & 1 & 0 & 0 & 1 & 1 & 0 & 0.64 \\
\hline Citrus reticulata & 1 & 1 & 0 & 1 & 1 & 0 & 1 & 0 & 1 & 1 & 0 & 0.64 \\
\hline Citrus aurantium & 1 & 0 & 1 & 1 & 0 & 1 & 0 & 1 & 1 & 0 & 0 & 0.55 \\
\hline Portulaca oleracea & 1 & 0 & 1 & 1 & 1 & 0 & 0 & 1 & 1 & 0 & 0 & 0.55 \\
\hline Rosmarinus officinalis & 1 & 0 & 0 & 0 & 1 & 0 & 0 & 0 & 1 & 1 & 1 & 0.45 \\
\hline Yucca treculeana & 1 & 0 & 0 & 1 & 1 & 0 & 0 & 0 & 1 & 0 & 1 & 0.45 \\
\hline Canavalia septentrionalis & 1 & 0 & 0 & 1 & 0 & 0 & 0 & 0 & 1 & 1 & 0 & 0.36 \\
\hline Eugenia capuli & 1 & 0 & 0 & 1 & 1 & 0 & 0 & 0 & 1 & 0 & 0 & 0.36 \\
\hline Asparagus officinalis & 0 & 0 & 0 & 0 & 1 & 0 & 0 & 0 & 0 & 1 & 0 & 0.18 \\
\hline $\mathrm{V}_{\mathrm{s}}$ & 1.17 & 0.96 & 0.93 & 1.02 & 1.04 & 0.91 & 0.87 & 0.83 & 1.13 & 0.83 & 0.69 & \\
\hline
\end{tabular}

$\mathrm{V}_{\mathrm{t}}=$ number of uses mentioned for each species by a given interviewee; $\mathrm{V}_{\mathrm{s}}=\Sigma \mathrm{V}_{\mathrm{t}} /$ number of species observed per interviewee; $\mathrm{V}_{\text {sppa }}=\Sigma \mathrm{V}_{\mathrm{t}} /$ number of adult male interviewees that observed the species; $\mathrm{V}_{\mathrm{ppa}}=$ $\Sigma \mathrm{Vs} /$ number of adult male interviewees. 
Table 3. Use value of food species in Las Guapas community, by species and interviewee group.

\begin{tabular}{|c|c|c|c|c|c|c|}
\hline Scientific name & Habitat & $\mathbf{V}_{\text {spma }}$ & $\mathbf{V}_{\text {sppa }}$ & $\mathbf{V}_{\text {spja }}$ & $\mathbf{V}_{\text {spjo }}$ & $\mathbf{V}_{\text {sptotal }}$ \\
\hline Erythrina coralloides & $\mathrm{H}$ & 1.48 & 2.73 & 1.06 & 2.00 & 1.66 \\
\hline Ficus cotinifolia & $\mathrm{H}, \mathrm{F}$ & 1.38 & 2.36 & 0.94 & 1.57 & 1.46 \\
\hline Persea americana & $\mathrm{H}, \mathrm{F}$ & 1.33 & 1.91 & 1.24 & 1.71 & 1.46 \\
\hline Helianthus annuus & $\mathrm{H}, \mathrm{CL}$ & 1.24 & 1.18 & 1.00 & 1.00 & 1.13 \\
\hline Chenopodium ambrosioides & $\mathrm{H}, \mathrm{CL}$ & 1.10 & 1.18 & 1.00 & 1.00 & 1.07 \\
\hline Carya ovata var. mexicana & $\mathrm{H}, \mathrm{F}$ & 1.14 & 1.00 & 1.00 & 1.00 & 1.05 \\
\hline Juglans mollis. & $\mathrm{H}, \mathrm{F}$ & 1.14 & 1.00 & 1.00 & 1.00 & 1.05 \\
\hline Ruta chalepensis & $\mathrm{H}$ & 1.14 & 1.00 & 1.00 & 1.00 & 1.05 \\
\hline Mangifera indica & $\mathrm{H}$ & 1.14 & 1.00 & 0.94 & 1.00 & 1.04 \\
\hline Psidium guajava & $\mathrm{H}$ & 1.00 & 1.18 & 1.00 & 1.00 & 1.04 \\
\hline Cnidosculus multilobus & $\mathrm{CL}, \mathrm{OL}, \mathrm{F}$ & 1.00 & 1.09 & 1.00 & 1.00 & 1.02 \\
\hline Musa $\mathrm{x}$ paradisiaca & $\mathrm{H}, \mathrm{CL}$ & 1.00 & 1.00 & 1.06 & 1.00 & 1.02 \\
\hline Amaranthus bybridus & $\mathrm{H}, \mathrm{CL}, \mathrm{OL}$ & 1.00 & 1.00 & 1.00 & 1.00 & 1.00 \\
\hline Carica papaya & $\mathrm{H}, \mathrm{CL}$ & 1.00 & 1.00 & 1.00 & 1.00 & 1.00 \\
\hline Gonolobus niger & $\mathrm{H}, \mathrm{F}$ & 1.00 & 1.00 & 1.00 & 1.00 & 1.00 \\
\hline Nopalea cochenillifera & $\mathrm{H}, \mathrm{CL}$ & 1.00 & 1.00 & 1.00 & 1.00 & 1.00 \\
\hline Opuntia sp. & $\mathrm{H}, \mathrm{CL}, \mathrm{OL}$ & 1.00 & 1.00 & 1.00 & 1.00 & 1.00 \\
\hline Curcuma longa & $\mathrm{H}, \mathrm{CL}$ & 1.00 & 0.91 & 1.00 & 1.00 & 0.98 \\
\hline Phaseolus coccineus & CL & 0.95 & 1.00 & 1.00 & 1.00 & 0.98 \\
\hline Phaseolus vulgaris & CL & 0.95 & 1.00 & 1.00 & 1.00 & 0.98 \\
\hline Vigna unguiculata & CL & 0.95 & 1.00 & 1.00 & 1.00 & 0.98 \\
\hline Coffea arabica & $\mathrm{H}, \mathrm{CL}, \mathrm{F}$ & 0.90 & 1.09 & 0.94 & 1.00 & 0.96 \\
\hline Conostegia xalapensis & $\mathrm{H}, \mathrm{CL}, \mathrm{OL}$ & 0.90 & 1.00 & 1.00 & 1.00 & 0.96 \\
\hline Saccharum officinarum & $\mathrm{H}, \mathrm{CL}$ & 0.95 & 1.00 & 0.94 & 1.00 & 0.96 \\
\hline Zingiber officinale & $\mathrm{H}, \mathrm{CL}$ & 1.00 & 1.00 & 0.88 & 1.00 & 0.96 \\
\hline & & 0.88 & 0.94 & 0.82 & 0.95 & \\
\hline & & & \multirow{2}{*}{\multicolumn{2}{|c|}{$\begin{array}{l}\mathbf{V}_{\mathbf{p}} \text { total women } \\
\mathbf{V}_{\mathrm{p}} \text { total men }\end{array}$}} & & 0.85 \\
\hline & & & & & & 0.95 \\
\hline
\end{tabular}

$\mathrm{V}_{\text {sppa }}=\Sigma \mathrm{V}_{\mathrm{t}} /$ number of adult male interviewees that observed the species. This also applies for $\mathrm{V}_{\text {spma }}=$ adult women; $\mathrm{V}_{\text {spjo }}=$ underaged men; $\mathrm{V}_{\text {spia }}=$ underaged women; $\mathrm{V}_{\mathrm{p}}=\Sigma \mathrm{V}_{\mathrm{s}} /$ number of interviewees in each group. Only 25 of the 54 species used in this investigation are shown (refer to Appendix 1). Habitat: $\mathrm{H}=$ homegarden, $\mathrm{CL}=$ cultivated land, $\mathrm{F}=$ forest, $\mathrm{OL}=$ other anthropic land (roadside, wasteland, etc.) 


\section{(A) Ethnobiolocr Letters}

\section{Research Communication}

Supplementary Table 1. Use value assigned to plants by interviewees in Las Guapas community.

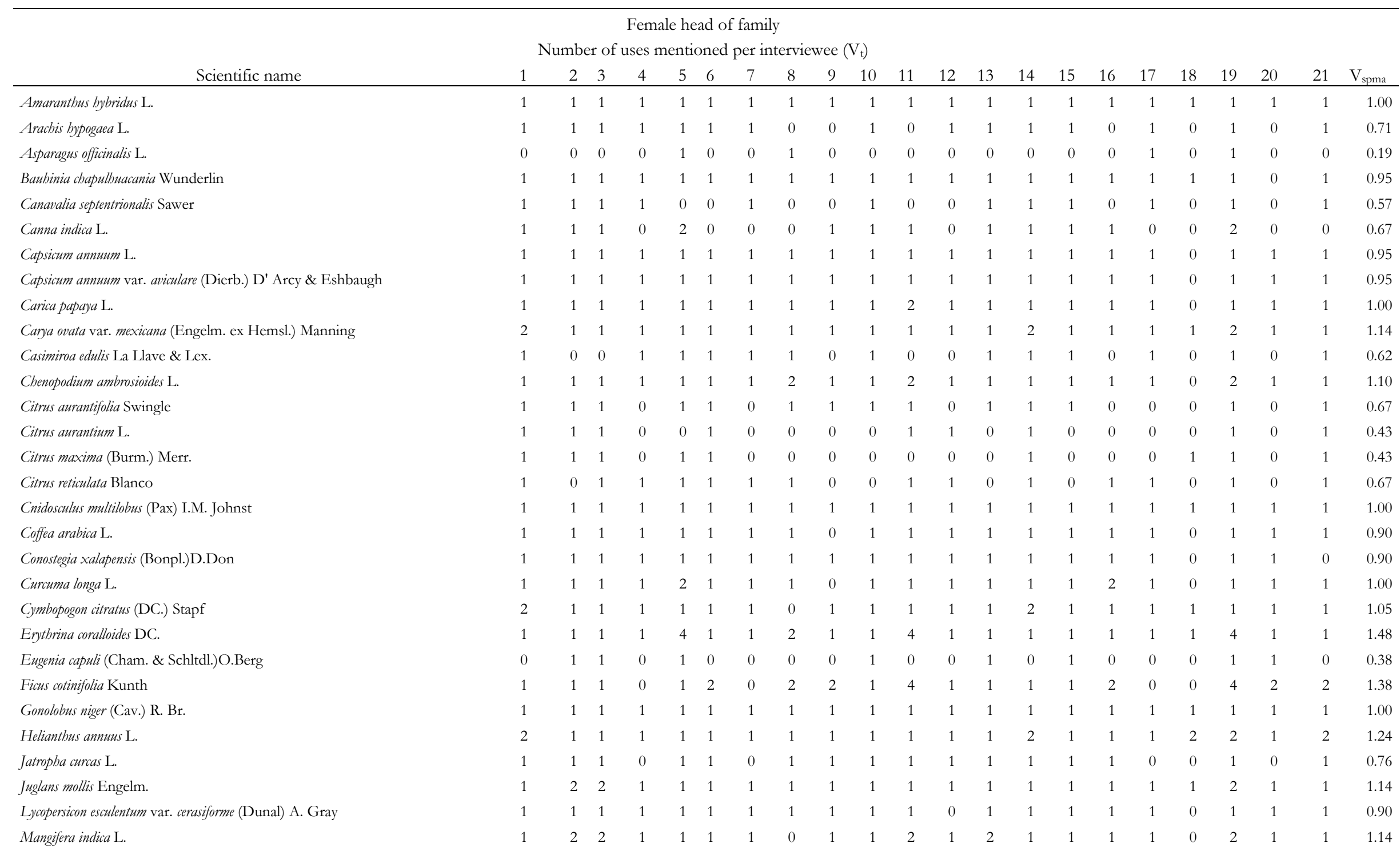




\section{(f) Ethnobiolocy Letters}

\section{Research Communication}

Manihot esculenta Crantz

Morus aff. celtidifolia Kunth

Musa $\mathrm{x}$ paradisiaca $\mathrm{L}$.

Nopalea cochenillifera (L.) Salm-Dyck

Opuntia

Pachyrbizus erosus (L.) Urb.

Persea americana Mill.

Phaseolus coccineus $\mathrm{L}$.

Phaseolus vulgaris $\mathrm{L}$.

Physalis philadelpbica Lam.

Phytolacca icosandra $\mathrm{L}$

Pisum sativum $\mathrm{L}$

Portulaca oleracea $\mathrm{L}$.

Psidium guajava L.

Rosmarinus officinalis $\mathrm{L}$.

Ruta chalepensis $\mathrm{L}$.

Saccharum officinarum $\mathrm{L}$.

Sechium edule (Jacq.) Sw.

Syngonium podophyllum Schott.

Tagetes filifolia Lag

Tigridia pavonia (L.f.) DC.

Vigna unguiculata (L.) Walp.

Yucca treculeana Carr.

Zingiber officinale Roscoe

\begin{tabular}{rrrrrrrrrrrrrrrrrrrrr}
1 & 1 & 0 & 1 & 1 & 0 & 1 & 1 & 1 & 1 & 1 & 1 & 1 & 1 & 1 & 0 & 1 & 1 & 0 & 1 & 0.81 \\
1 & 1 & 1 & 1 & 1 & 1 & 0 & 0 & 1 & 0 & 1 & 1 & 0 & 1 & 0 & 1 & 0 & 1 & 0 & 0 & 0.57 \\
1 & 1 & 1 & 1 & 1 & 1 & 1 & 1 & 1 & 1 & 1 & 1 & 1 & 1 & 1 & 1 & 1 & 1 & 1 & 1 & 1.00 \\
1 & 1 & 1 & 1 & 1 & 1 & 1 & 1 & 1 & 1 & 1 & 1 & 1 & 1 & 1 & 1 & 1 & 1 & 1 & 1 & 1.00 \\
1 & 1 & 1 & 1 & 1 & 1 & 1 & 1 & 1 & 1 & 1 & 1 & 1 & 1 & 1 & 1 & 1 & 1 & 1 & 1 & 1.00 \\
1 & 1 & 1 & 1 & 0 & 1 & 0 & 1 & 1 & 1 & 1 & 1 & 1 & 1 & 1 & 1 & 0 & 1 & 1 & 1 & 0.86 \\
1 & 1 & 1 & 1 & 3 & 1 & 1 & 2 & 1 & 1 & 1 & 2 & 1 & 1 & 2 & 1 & 0 & 3 & 2 & 1 & 1.33 \\
1 & 1 & 1 & 1 & 1 & 1 & 1 & 1 & 1 & 1 & 1 & 1 & 1 & 1 & 1 & 1 & 0 & 1 & 1 & 1 & 0.95 \\
1 & 1 & 1 & 1 & 1 & 1 & 1 & 1 & 1 & 1 & 1 & 1 & 1 & 1 & 1 & 1 & 0 & 1 & 1 & 1 & 0.95 \\
1 & 1 & 0 & 2 & 1 & 0 & 1 & 0 & 1 & 0 & 1 & 1 & 0 & 1 & 0 & 0 & 0 & 1 & 1 & 1 & 0.62 \\
1 & 1 & 1 & 1 & 1 & 1 & 1 & 0 & 1 & 1 & 1 & 1 & 1 & 1 & 0 & 1 & 0 & 1 & 0 & 1 & 0.81 \\
1 & 1 & 1 & 1 & 1 & 1 & 1 & 1 & 1 & 1 & 1 & 1 & 1 & 1 & 1 & 1 & 0 & 1 & 0 & 1 & 0.90 \\
1 & 1 & 1 & 1 & 1 & 1 & 0 & 0 & 1 & 0 & 1 & 1 & 0 & 1 & 0 & 1 & 0 & 1 & 0 & 1 & 0.62 \\
1 & 2 & 1 & 1 & 1 & 1 & 1 & 1 & 1 & 1 & 1 & 1 & 1 & 1 & 1 & 1 & 0 & 1 & 1 & 1 & 1.00 \\
1 & 1 & 1 & 1 & 1 & 1 & 1 & 1 & 1 & 0 & 1 & 1 & 1 & 1 & 1 & 1 & 1 & 1 & 0 & 1 & 0.90 \\
1 & 1 & 1 & 1 & 1 & 1 & 1 & 1 & 2 & 1 & 1 & 2 & 1 & 2 & 1 & 1 & 0 & 2 & 1 & 1 & 1.14 \\
1 & 1 & 1 & 1 & 1 & 1 & 1 & 1 & 1 & 1 & 1 & 1 & 1 & 1 & 1 & 1 & 0 & 1 & 1 & 1 & 0.95 \\
1 & 1 & 1 & 1 & 1 & 1 & 0 & 0 & 1 & 1 & 1 & 1 & 1 & 1 & 1 & 1 & 0 & 1 & 1 & 0 & 0.81 \\
1 & 1 & 1 & 1 & 1 & 1 & 1 & 1 & 1 & 1 & 0 & 1 & 0 & 1 & 0 & 1 & 0 & 1 & 0 & 1 & 0.71 \\
1 & 1 & 1 & 1 & 1 & 1 & 1 & 1 & 1 & 1 & 1 & 1 & 1 & 1 & 1 & 1 & 0 & 1 & 1 & 1 & 0.95 \\
1 & 1 & 1 & 1 & 1 & 1 & 1 & 0 & 1 & 1 & 0 & 1 & 1 & 1 & 0 & 1 & 0 & 1 & 0 & 1 & 0.76 \\
1 & 1 & 1 & 1 & 1 & 1 & 1 & 1 & 1 & 1 & 1 & 1 & 1 & 1 & 1 & 1 & 0 & 1 & 1 & 1 & 0.95 \\
0 & 0 & 0 & 1 & 1 & 0 & 0 & 1 & 1 & 0 & 1 & 1 & 1 & 1 & 1 & 0 & 0 & 1 & 0 & 0 & 0.48 \\
2 & 2 & 1 & 2 & 2 & 1 & 1 & 0 & 1 & 1 & 0 & 1 & 1 & 1 & 0 & 1 & 0 & 2 & 0 & 1 & 1.00 \\
\hline 0.98 & 1 & 0.8 & 1.1 & 1 & 0.8 & 0.8 & 0.7 & 0.9 & 1 & 0.8 & 1 & 0.9 & 0.9 & 0.8 & 0.8 & 0.3 & 1.3 & 0.6 & 0.91 & 0.88 \\
& & & & & & & & & & & & & & & & & & $V_{\text {pma }}$ & 0.90101
\end{tabular}

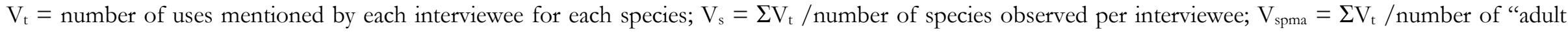
women" interviewees that observed the species; $\mathrm{V}_{\mathrm{pma}}=\Sigma \mathrm{V}_{\mathrm{s}} /$ number of "adult women" interviewees. 


\section{(f) Ethnobiolocy Letters}

\section{Research Communication}

Supplementary Table 1. (Cont.).

\begin{tabular}{|c|c|c|c|c|c|c|c|c|c|c|c|c|c|c|c|c|c|c|c|c|}
\hline \multirow[b]{2}{*}{ Scientific name } & \multicolumn{10}{|c|}{ Male head of family } & \multicolumn{10}{|c|}{$\begin{array}{c}\text { Underage sons ( } \geq 12 \leq 22 \text { years old }) \\
\text { Number of uses mentioned per interviewee }\left(\mathrm{V}_{\mathrm{t}}\right)\end{array}$} \\
\hline & 1 & 2 & 3 & 4 & 5 & 6 & 7 & 8 & 9 & 10 & 11 & $V_{\text {sppa }}$ & 1 & 2 & 3 & 4 & 5 & 6 & 7 & $V_{\text {spjo }}$ \\
\hline Amaranthus hybridus L. & 1 & 1 & 1 & 1 & 1 & 1 & 1 & 1 & 1 & 1 & 1 & 1.00 & 1 & 1 & 1 & 1 & 1 & 1 & 1 & 1.00 \\
\hline Arachis bypogaea $\mathrm{L}$. & 1 & 1 & 1 & 1 & 1 & 0 & 1 & 1 & 1 & 0 & 0 & 0.73 & 1 & 1 & 1 & 1 & 1 & 1 & 1 & 1.00 \\
\hline Asparagus officinalis $\mathrm{L}$. & 0 & 0 & 0 & 0 & 1 & 0 & 0 & 0 & 0 & 1 & 0 & 0.18 & 0 & 0 & 0 & 0 & 0 & 0 & 0 & 0.00 \\
\hline Baubinia chapulbuacania Wunderlin & 1 & 1 & 1 & 1 & 1 & 1 & 1 & 1 & 1 & 1 & 1 & 1.00 & 0 & 1 & 1 & 1 & 1 & 1 & 1 & 0.86 \\
\hline Canavalia septentrionalis Sawer & 1 & 0 & 0 & 1 & 0 & 0 & 0 & 0 & 1 & 1 & 0 & 0.36 & 0 & 0 & 1 & 1 & 1 & 1 & 1 & 0.71 \\
\hline Canna indica $\mathrm{L}$. & 1 & 1 & 2 & 1 & 1 & 0 & 1 & 1 & 1 & 0 & 1 & 0.91 & 0 & 1 & 1 & 1 & 1 & 1 & 1 & 0.86 \\
\hline Capsicum anпuиm $\mathrm{L}$. & 1 & 0 & 1 & 1 & 1 & 1 & 0 & 1 & 1 & 1 & 1 & 0.82 & 1 & 1 & 1 & 1 & 1 & 1 & 1 & 1.00 \\
\hline Capsicum annuum var. aviculare (Dierb.) D' Arcy \& Eshbaugh & 1 & 0 & 1 & 1 & 1 & 1 & 0 & 1 & 1 & 1 & 1 & 0.82 & 1 & 1 & 1 & 1 & 1 & 1 & 1 & 1.00 \\
\hline Carica papaya $\mathrm{L}$. & 1 & 1 & 1 & 1 & 1 & 1 & 1 & 1 & 1 & 1 & 1 & 1.00 & 1 & 1 & 1 & 1 & 1 & 1 & 1 & 1.00 \\
\hline Carya ovata var. mexicana (Engelm. ex Hemsl.) Manning & 1 & 1 & 1 & 1 & 1 & 1 & 1 & 1 & 1 & 1 & 1 & 1.00 & 1 & 1 & 1 & 1 & 1 & 1 & 1 & 1.00 \\
\hline Casimiroa edulis La Llave \& Lex. & 1 & 1 & 0 & 1 & 1 & 0 & 0 & 0 & 1 & 1 & 1 & 0.64 & 0 & 1 & 1 & 1 & 1 & 1 & 1 & 0.86 \\
\hline Chenopodium ambrosioides $\mathrm{L}$. & 1 & 1 & 1 & 1 & 1 & 2 & 1 & 1 & 1 & 2 & 1 & 1.18 & 1 & 1 & 1 & 1 & 1 & 1 & 1 & 1.00 \\
\hline Citrus aurantifolia Swingle & 1 & 1 & 1 & 1 & 0 & 1 & 0 & 0 & 1 & 1 & 0 & 0.64 & 0 & 1 & 1 & 0 & 1 & 0 & 1 & 0.57 \\
\hline Citrus aurantium $\mathrm{L}$. & 1 & 0 & 1 & 1 & 0 & 1 & 0 & 1 & 1 & 0 & 0 & 0.55 & 0 & 1 & 1 & 1 & 1 & 1 & 1 & 0.86 \\
\hline Citrus maxima (Burm.) Merr. & 1 & 1 & 1 & 1 & 1 & 0 & 1 & 1 & 1 & 0 & 0 & 0.73 & 1 & 0 & 1 & 1 & 1 & 1 & 1 & 0.86 \\
\hline Citrus reticulata Blanco & 1 & 1 & 0 & 1 & 1 & 0 & 1 & 0 & 1 & 1 & 0 & 0.64 & 0 & 0 & 1 & 1 & 1 & 0 & 1 & 0.57 \\
\hline Cnidosculus multilobus (Pax) I.M. Johnst & 2 & 1 & 1 & 1 & 1 & 1 & 1 & 1 & 1 & 1 & 1 & 1.09 & 1 & 1 & 1 & 1 & 1 & 1 & 1 & 1.00 \\
\hline Coffea arabica $\mathrm{L}$. & 1 & 2 & 1 & 1 & 1 & 1 & 2 & 1 & 1 & 1 & 0 & 1.09 & 1 & 1 & 1 & 1 & 1 & 1 & 1 & 1.00 \\
\hline Conostegia xalapensis (Bonpl.)D.Don & 1 & 1 & 1 & 1 & 1 & 1 & 1 & 1 & 1 & 1 & 1 & 1.00 & 1 & 1 & 1 & 1 & 1 & 1 & 1 & 1.00 \\
\hline Curcuma longa $\mathrm{L}$. & 1 & 1 & 1 & 1 & 1 & 1 & 1 & 1 & 1 & 1 & 0 & 0.91 & 1 & 1 & 1 & 1 & 1 & 1 & 1 & 1.00 \\
\hline Cymbopogon citratus (DC.) Stapf & 1 & 1 & 1 & 1 & 1 & 1 & 1 & 1 & 1 & 0 & 1 & 0.91 & 0 & 0 & 1 & 1 & 1 & 1 & 1 & 0.71 \\
\hline Erythrina coralloides DC. & 4 & 3 & 2 & 2 & 4 & 4 & 3 & 1 & 4 & 2 & 1 & 2.73 & 1 & 1 & 2 & 3 & 2 & 2 & 3 & 2.00 \\
\hline Eugenia capuli (Cham. \& Schltdl.)O.Berg & 1 & 0 & 0 & 1 & 1 & 0 & 0 & 0 & 1 & 0 & 0 & 0.36 & 1 & 1 & 1 & 1 & 1 & 1 & 1 & 1.00 \\
\hline Ficus cotinifolia Kunth & 3 & 4 & 2 & 2 & 1 & 4 & 3 & 2 & 2 & 2 & 1 & 2.36 & 0 & 1 & 2 & 2 & 2 & 2 & 2 & 1.57 \\
\hline Gonolobus niger (Cav.) R. Br. & 1 & 1 & 1 & 1 & 1 & 1 & 1 & 1 & 1 & 1 & 1 & 1.00 & 1 & 1 & 1 & 1 & 1 & 1 & 1 & 1.00 \\
\hline Helianthus anпuиs $\mathrm{L}$. & 2 & 2 & 1 & 1 & 1 & 1 & 1 & 1 & 1 & 1 & 1 & 1.18 & 1 & 1 & 1 & 1 & 1 & 1 & 1 & 1.00 \\
\hline Jatropha curcas $\mathrm{L}$. & 1 & 1 & 0 & 1 & 1 & 1 & 1 & 0 & 1 & 1 & 1 & 0.82 & 0 & 1 & 1 & 1 & 1 & 1 & 1 & 0.86 \\
\hline Juglans mollis Engelm. & 1 & 1 & 1 & 1 & 1 & 1 & 1 & 1 & 1 & 1 & 1 & 1.00 & 1 & 1 & 1 & 1 & 1 & 1 & 1 & 1.00 \\
\hline Lycopersicon esculentum var. cerasiforme (Dunal) A. Gray & 1 & 1 & 1 & 1 & 1 & 1 & 1 & 1 & 1 & 1 & 1 & 1.00 & 1 & 1 & 1 & 1 & 1 & 1 & 1 & 1.00 \\
\hline Mangifera indica $\mathrm{L}$. & 1 & 1 & 1 & 1 & 1 & 2 & 1 & 1 & 1 & 0 & 1 & 1.00 & 1 & 1 & 1 & 1 & 1 & 1 & 1 & 1.00 \\
\hline
\end{tabular}




\section{(f) Ethnobiolocy Letters}

Research Communication

Manibot esculenta Crantz

Morus aff. celtidifolia Kunth

Musa $\times$ paradisiaca L.

Nopalea cochenillifera (L.) Salm-Dyck

Opuntia

Pachyrbizus erosus (L.) Urb.

Persea americana Mill.

Phaseolus coccineus $\mathrm{L}$.

Phaseolus vulgaris $\mathrm{L}$.

Physalis pbiladelpbica Lam.

Phytolacca icosandra L.

Pisum sativum $\mathrm{L}$.

Portulaca oleracea $\mathrm{L}$.

Psidium guajava L.

Rosmarinus officinalis $\mathrm{L}$.

Ruta chalepensis $\mathrm{L}$.

Saccharum officinarum $\mathrm{L}$.

Secbium edule (Jacq.) Sw.

Syngonium podophyllum Schott.

Tagetes filifolia Lag

Tigridia pavonia (L.f.) DC.

Vigna unguiculata (L.) Walp.

Yucca treculeana Carr.

Zingiber officinale Roscoe

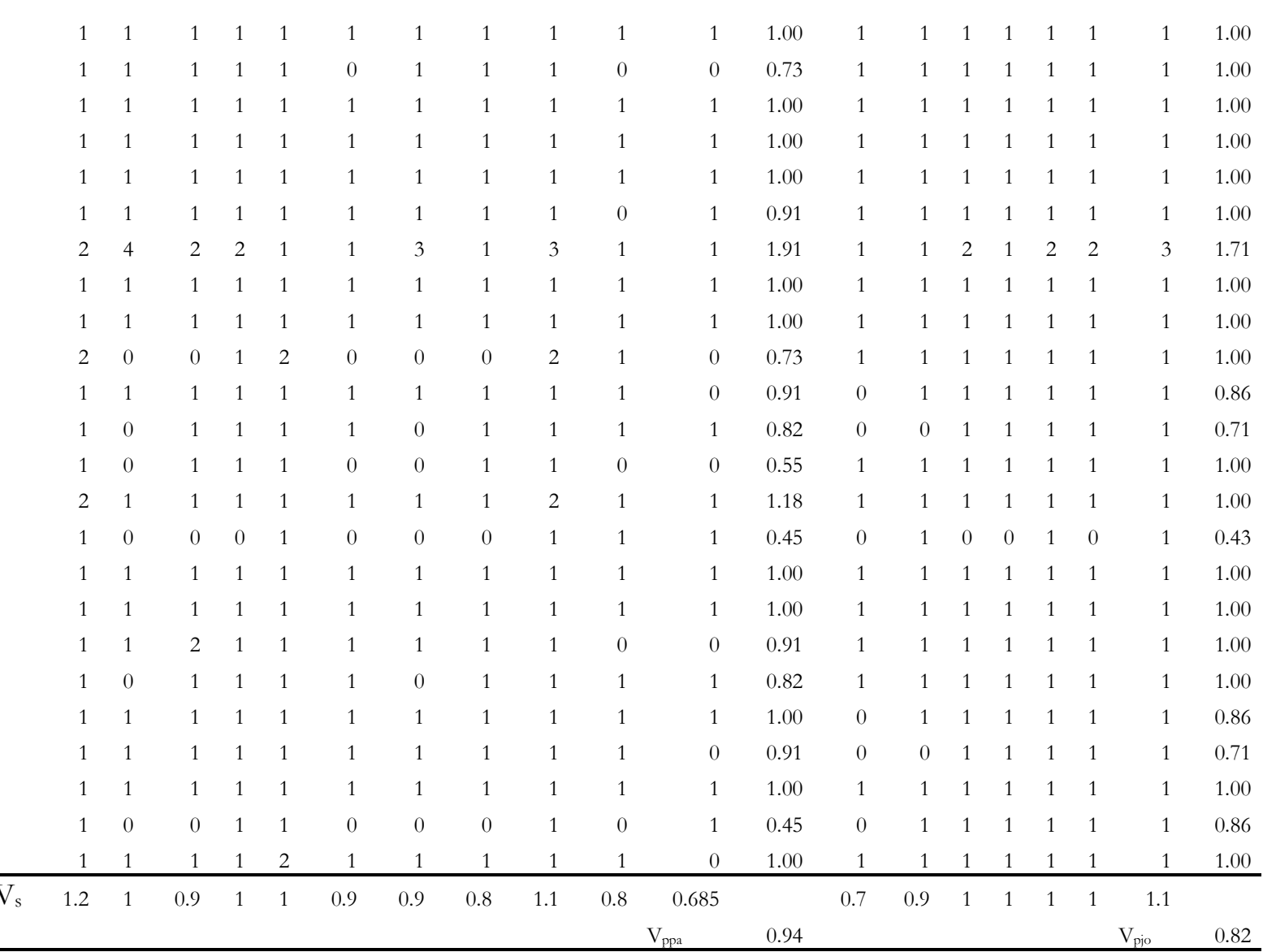

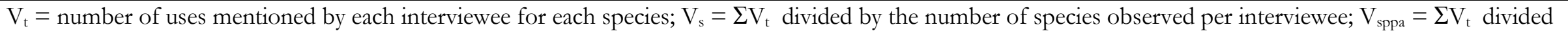

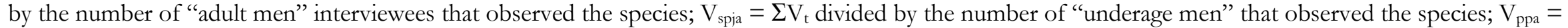
$\Sigma \mathrm{V}_{\mathrm{s}}$ divided by the number of "adult men" interviewees; $\mathrm{V}_{\mathrm{pjo}}=\Sigma \mathrm{V}_{\mathrm{s}}$ divided by the number of “ underage men" interviewees. 


\section{(f) Ethnobiolocy Letters}

\section{Research Communication}

Supplementary Table 1. (Cont.).

Single daughters ( $\geq 12 \leq 22$ years old $)$

Number of uses mentioned per interviewee $\left(V_{t}\right)$

Amaranthus hybridus $\mathrm{L}$

Arachis hypogaea $\mathrm{L}$.

Asparagus officinalis $\mathrm{L}$.

Baubinia chapulbuacania Wunderlin

Canavalia septentrionalis Sawer

Canna indica $\mathrm{L}$.

Capsicum annuum $\mathrm{L}$.

Capsicum annum var. aviculare (Dierb.) D' Arcy \& Eshbaugh

\section{Carica papaya $\mathrm{L}$.}

Carya ovata var. mexicana (Engelm. ex Hemsl.) Manning

Casimiroa edulis La Llave \& Lex.

Chenopodium ambrosioides $\mathrm{L}$.

Citrus aurantifolia Swingle

Citrus aurantium $\mathrm{L}$.

Citrus maxima (Burm.) Merr.

Citrus reticulata Blanco

Cnidosculus multilobus (Pax) I.M. Johnst

\section{Coffea arabica $\mathrm{L}$.}

Conostegia xalapensis (Bonpl.)D.Don

Curcuma longa $\mathrm{L}$.

Cymbopogon citratus (DC.) Stapf

Erythrina coralloides DC.

Eugenia capuli (Cham. \& Schltdl.)O.Berg

Ficus cotinifolia Kunth

Gonolobus niger (Cav.) R. Br.

Helianthus annuиs $\mathrm{L}$.

Jatropha curcas L.

Juglans mollis Engelm.

Lycopersicon esculentum var. cerasiforme (Dunal) A. Gray

Mangifera indica $\mathrm{L}$.

4

$5 \quad 6$
1

$\begin{array}{rrrrrrrrr}6 & 7 & 8 & 9 & 10 & 11 & 12 & 13 \\ 1 & 1 & 1 & 1 & 1 & 1 & 1 & 1 & 1\end{array}$

$\begin{array}{rrr}15 & 16 \\ 1 & 1\end{array}$

11.00

0

$\begin{array}{llllllllllll}1 & 0 & 0 & 0 & 0 & 0 & 0 & 0 & 0 & 1 & 0 & 0.18\end{array}$




\section{(f) Ethnobiolocy Letters}

\section{Research Communication}

Manibot esculenta Crantz

Morus aff. celtidifolia Kunth

Musa x paradisiaca L.

Nopalea cochenillifera (L.) Salm-Dyck

\section{Opuntia}

Pachyrbizus erosus (L.) Urb.

Persea americana Mill.

Phaseolus coccineus $\mathrm{L}$.

Phaseolus vulgaris $\mathrm{L}$.

Physalis philadelphica Lam.

Phytolacca icosandra $\mathrm{L}$.

Pisum sativum $\mathrm{L}$.

Portulaca oleracea $\mathrm{L}$.

Psidium guajava $\mathrm{L}$.

Rosmarinus officinalis $\mathrm{L}$.

Ruta chalepensis $\mathrm{L}$.

Saccharum officinarum $\mathrm{L}$.

Secbium edule (Jacq.) Sw.

Syngonium podophyllum Schott.

Tagetes filifolia Lag

Tigridia pavonia (L.f.) DC.

Vigna unguiculata (L.) Walp.

Yucca treculeana Carr.

Zingiber officinale Roscoe

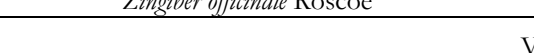

Vs 0.8




\section{(f) Ethnobiolocr Letters}

\section{Research Communication}

Supplementary Table 2 . List of food species referred to in the text.

\begin{tabular}{|c|c|c|c|}
\hline Scientific name & Family & Spanish name & $X i^{\prime} i u y$ name* \\
\hline Amaranthus bybridus $\mathrm{L}$. & Amaranthaceae & Quelite & xixium \\
\hline Arachis bypogaea $\mathrm{L}$. & Fabaceae & Cacahuate & ampogose/dempogose \\
\hline Asparagus officinalis $\mathrm{L}$. & Liliaceae & Espárrago & \\
\hline Baubinia chapulbuacania Wunderlin & Fabaceae & Pata de vaca & vacua pagas/shankuc \\
\hline Canavalia septentrionalis Sawer & Fabaceae & Conchito & cujuel/gaun \\
\hline Canna indica $\mathrm{L}$. & Cannaceae & Platanillo & vin ob \\
\hline Capsicum anпuиm $\mathrm{L}$. & Solanaceae & Chile pico de pájaro & ilju shinyua shiljai \\
\hline Capsicum annuum var. aviculare (Dierb.) D' Arcy \& Eshbaugh & Solanaceae & Chile piquín & ilju quipin \\
\hline Carica papaya L. & Caricaceae & Papaya & \\
\hline Carya ovata var. mexicana (Engelm. ex Hemsl.) Manning & Juglandaceae & Nogal & jusé/ gatun \\
\hline Casimiroa edulis La Llave \& Lex. & Rutaceae & Zapote blanco & rapot denua \\
\hline Chenopodium ambrosioides $\mathrm{L}$. & Chenopodiaceae & Epazote & shquipis \\
\hline Citrus aurantifolia Swingle & Rutaceae & Limón dulce y limón agrío & danaas vaas/ danaas vais \\
\hline Citrus aurantium $\mathrm{L}$. & Rutaceae & Naranja cucha & danaas vais \\
\hline Citrus maxima (Burm.) Merr. & Rutaceae & Toronja & danaas vaas \\
\hline Citrus reticulata Blanco & Rutaceae & Mandarina & danaas vaus \\
\hline Cnidosculus multilobus (Pax) I.M. Johnst & Euphorbiaceae & Mala mujer & xkete \\
\hline Coffea arabica $\mathrm{L}$. & Rubiaceae & Café & kepiai \\
\hline Conostegia xalapensis (Bonpl.)D.Don & Melastomataceae & Garambullo & ximpion \\
\hline Curcuma longa $\mathrm{L}$. & Zingiberaceae & Azafrán & miyuandajuan \\
\hline Cymbopogon citratus (DC.) Stapf & Poaceae & Zacate limón & danaas insu/danaas sansu \\
\hline Erytbrina coralloides DC. & Fabaceae & Patol & ndaá \\
\hline Eugenia capuli (Cham. \& Schltdl.)O.Berg & Myrtaceae & Capulín & datuen \\
\hline Ficus cotinifolia Kunth & Moraceae & Higuerón & \\
\hline Gonolobus niger (Cav.) R. Br. & Asclepiadaceae & Talayote & gajú \\
\hline Helianthus annuиs $\mathrm{L}$. & Asteraceae & Girasol, gordolobo, maíz de teja & vinchin \\
\hline
\end{tabular}




\section{(f) Ethnobiolocy Letters}

\section{Research Communication}

\begin{tabular}{|c|c|c|c|}
\hline Scientific name & Family & Spanish name & Xi'iuy name* \\
\hline Jatropha curcas $\mathrm{L}$. & Euphorbiaceae & Pipián & góse \\
\hline Juglans mollis Engelm. & Juglandaceae & Nuez/ nogal & guse \\
\hline Lycopersicon esculentum var. cerasiforme (Dunal) A. Gray & Solanaceae & Tomate coyol & spail dapai nacua \\
\hline Mangifera indica $\mathrm{L}$. & Anacardiaceae & Mango corazón de burro, mango manila y mango corriente & \\
\hline Manibot esculenta Crantz & Euphorbiaceae & Yuca & \\
\hline Morus aff. celtidifolia Kunth & Moraceae & Mora & nkuan encush \\
\hline Musa $\mathrm{x}$ paradisiaca $\mathrm{L}$. & Musaceae & Plátano roatán & ntaas \\
\hline Nopalea cochenillifera (L.) Salm-Dyck & Cactaceae & Nopalito del huerto & mbiu \\
\hline Opuntia sp. & Cactaceae & Nopal manso & mbiu \\
\hline Pacbyrbizus erosus (L.) Urb. & Fabaceae & Jícama & minyuan \\
\hline Persea americana Mill. & Lauraceae & Aguacatillo, aguacate de monte, pagua, aguacate & nxaun \\
\hline Phaseolus coccineus $\mathrm{L}$. & Fabaceae & Frijol grande & kiet chiat \\
\hline Phaseolus vulgaris $\mathrm{L}$. & Fabaceae & Frijol ojo de conejo, frijol de mata, frijol de guia & chiant \\
\hline Physalis philadelpbica Lam. & Solanaceae & Tomate de bolsa, tomatillo de monte & indapuai lamuisemjul \\
\hline Phytolacca icosandra $\mathrm{L}$. & Phytolaccaceae & Congara, congora, conga, quelite hoja ancha, quelite punta colorada & esshauel kiljus \\
\hline Pisum sativum $\mathrm{L}$. & Fabaceae & Garbanzo & \\
\hline Portulaca oleracea L. & Portulacaceae & Verdolaga de adorno & sanke \\
\hline Psidium guajava $\mathrm{L}$. & Myrtaceae & Guayaba & buanjua/genjua \\
\hline Rosmarinus officinalis $\mathrm{L}$. & Lamiaceae & Romero & \\
\hline Ruta chalepensis $\mathrm{L}$. & Rutaceae & Ruda & \\
\hline Saccharum officinarum $\mathrm{L}$. & Poaceae & Caña blanca, caña borrada y caña morada & xiljua \\
\hline Sechium edule (Jacq.) Sw. & Cucurbitaceae & Chayote & datúa \\
\hline Syngonium podophyllum Schott. & Araceae & Huevo de burro & rinchu enmep/indkui mai \\
\hline Tagetes filifolia Lag & Asteraceae & Hierbanis, anis & \\
\hline Tigridia pavonia (L.f.) DC. & Iridaceae & Flor de calabaza, / carcoma/ oreja de perro & xcamoo \\
\hline Vigna unguiculata (L.) Walp. & Fabaceae & Chícharo & inyun chicbil \\
\hline Yucca treculeana Carr. & Agavaceae & Samandoque & ximbia \\
\hline Zingiber officinale Roscoe & Zingiberaceae & Jengibre & minyuan jengibre \\
\hline
\end{tabular}

